\title{
LAGO Ecuador, Implementing a set of WCD detectors for Space Weather research: first results and further developments
}

\author{
Stephany Vargas ${ }^{1 *}$, Cristina Mantilla ${ }^{1}$, Oscar Martinez ${ }^{1}$, Nicolás Vásquez ${ }^{1}$ and \\ Dennis Cazar $^{2}$ for the LAGO Collaboration ${ }^{3}$ \\ ${ }^{1}$ Escuela Politecnica Nacional, Departamento de Fisica, Ladron de Guevara E11-258, Quito, \\ Ecuador \\ ${ }^{2}$ Colegio de Ciencias e Ingenierías, Diego de Robles y Pampite S/N, Quito, Ecuador \\ ${ }^{3}$ http://lagoproject.org, see the full list of members and institutions at lagoproject.org/collab.html \\ E-mail:lago-pi@lagoproject.org \\ E-mail: stephany.vargas@epn.edu.ec, cristina.mantilla@epn.edu.ec
}

\begin{abstract}
The Latin American Giant Observatory (LAGO) is an international collaboration focused on the study of the phenomenology of Cosmic Rays (CR) in different energy ranges. Data is retrieved using Water Cherenkov Detectors(WCD) and by the execution of several simulations. Ecuador has been working in the LAGO project for almost 3 years, during this period three detectors had been installed in different universities of the country: one in Riobamba, ESPOCH (2784 m.a.s.l) and two in the city of Quito, USFQ (2430 m.a.s.1) and EPN (2830 m.a.s.l). A complete set of simulations have been performed within the LAGO simulation framework, based on a combination of Magnetocosmics, CORSIKA and GEANT4 codes. In this work, the detector characterization, calibration and the first Ecuadorian data is presented.
\end{abstract}

The 34th International Cosmic Ray Conference,

30 July- 6 August, 2015

The Hague, The Netherlands

${ }^{*}$ Speaker. 


\section{Background}

The Latin American Giant Observatory_LAGO_project is a recent organization created on 2005 [1]. There are 10 Latin American countries participating in this project, with more than 80 scientists and students of different disciplines [1]. As objectives, among others, this project studies high energy component of GRBs, transitory events, long term events, space weather and climate. These objectives are accomplished through the detection of the high energy component in GRBs for places located above 4000 meters over the sea level, through the study of solar modulation, the surveillance of the cosmic ray flow as well as other analysis. Academically, the project focuses on the study of astroparticle physics for undergraduate students including analysis and data statistics, detector's physics among other specific topics.
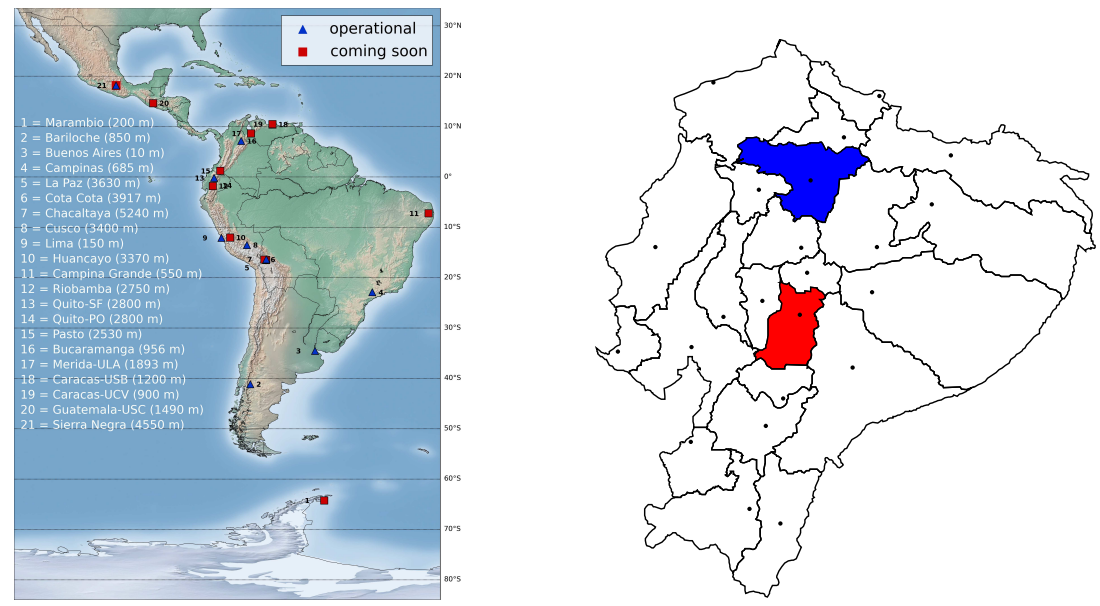

Figure 1: Left: LAGO locations, where blue triangles show the places associated with functional sites and red squares show the places that will work in 2015 [2]. Right: Political map of Ecuador. In red is the Chimborazo province where the "Chimbito" detector is located and in blue is the Pichincha Province where "Panchito" detector and "Politanque" detector are located.

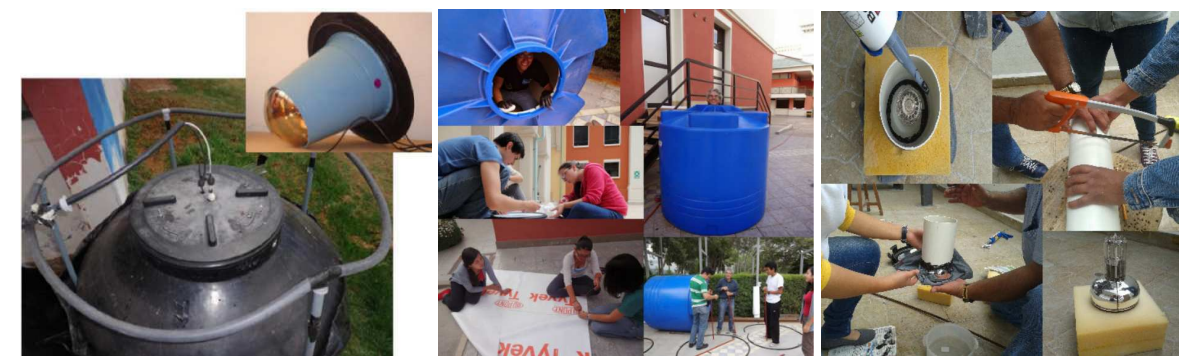

Figure 2: Left: "Chimbito" detector located in the province of Chimborazo, Center: construction of the "Panchito" detector located in the province of Pichincha in the valley of Cumbaya, Right: construction of the "Politanque" detector located in the province of Pichincha in Quito.

The LAGO network is unique considering its location (60 and 80 meridians perpendicular to the equatorial plane) and its extension through the southern hemisphere of the globe. The advantage of these characteristics is the possibility to register with great detail transit solar events, such as Coronal Mass Ejections (CME) simultaneously in several sites with very different rigidity cut-offs. 
The LAGO project in Ecuador began on 2012 at the Chimborazo Province, Ecuador [3]. The program started with an initial test on August and September at the ESPOCH University Campus with a detector named "Chimbito" [3]. On February 2014 at the USFQ University, located in the Pichincha Province, the construction of a second detector began, its was named "Panchito". Finally, a third detector, "Politanque", was implemented at the EPN University in Quito.

\section{Experimental setup and data}

The detector "Politanque" installed at the Escuela Politécnica Nacional (EPN), is the prototype for the Lago-Ecuador collaboration. Is a small-sized detector installed in the facilities of the University, accessible to students and teachers from within the university as well as other scientists within the collaboration. Its aim is to serve as a developing detector set up. "Politanque" has been fully operational for a short period of time.
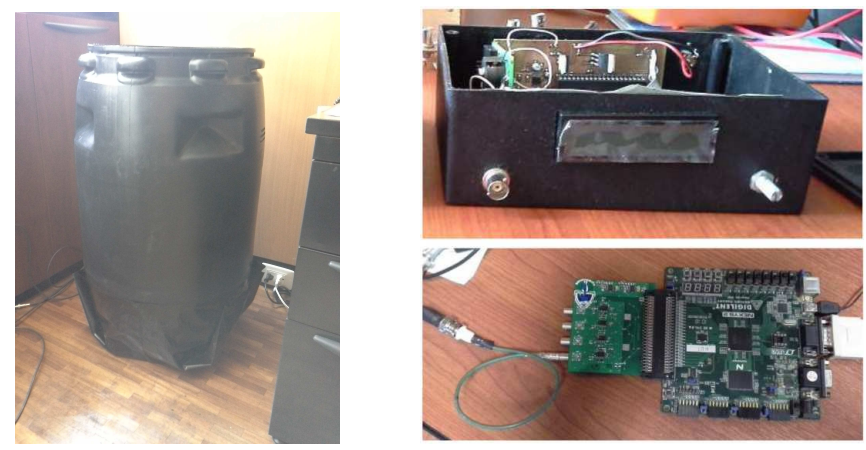

Figure 3: Left: Polyethylene-WCD, Rigth up: high-voltage source, Right down: DAQmx and Nexys2 Board, the electronics used for the experiment are from the EAS-BUAP project [4,5].

\subsection{WCD "Politanque"}

The detector consists of a black polyethylene tank that is $0.70[\mathrm{~m}]$ high with a radius $0.23[\mathrm{~m}]$ and with a volume of $0.11 \mathrm{~m}^{3}$. A 5" EMI 9530A photomultiplier is used. The high-voltage source, as well as the hardware and software used for data acquisition are from the EAS-BUAP project [5]. Data acquisition was performed using a voltage input $\sim 1450[V]$ for the PMT. The output signal is collected in a digitizer board-DAQmx (based on Dual ADC AD9216-100MS/s, 10bit resolution, 4-channel input), the information is then processed by a Nexys2 Board programed using VHDL, then it is stored and processed in a PC with Ubuntu 12.04.

\subsection{Data acquisition}

The USB-Serial Port connection from the preconfigured Nexys2 Board to the computer allows us to manipulate, via VHDL programing, the thresholds of the sampled data. The acquired data is in ADC counts in the range of nanoseconds. Each pulse has 16 samples of 10 nanosecond time length. 


\subsection{Data analysis}

We present the preliminary results of the data analysis of the Charge Distribution Histogram $(\mathrm{CDH})$ for a sample of 350000 events, this results are presented in figure 4 . We determined the VEM by a Gaussian fit from with we have a value of $456 \pm 106$ [pC]. In figure 5, we have the zoom of figure 4 from with we get the VEM and its Gaussian fit.

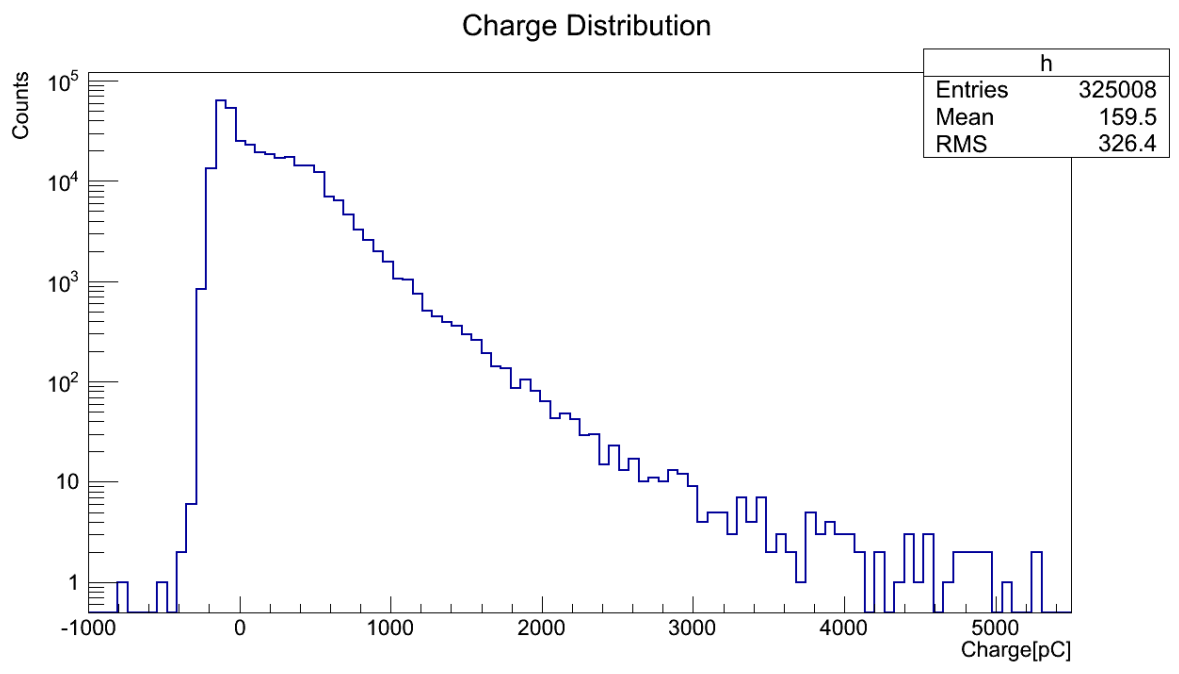

Figure 4: Charge Distribution Histogram (CDH) for "Politanque" detector.

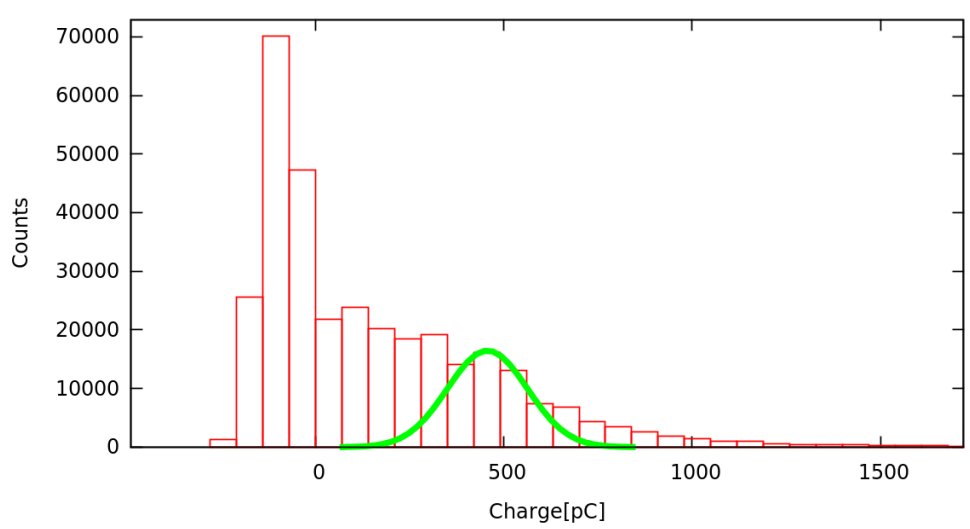

Figure 5: VEM (Vertical Equivalent Muon) adjusted by a Gaussian fit. We found a value of 456 [pC] with a statistical error of 106 .

\section{Simulation}

The data used in the Collaboration include data registered in Cherenkov detectors and data obtained through simulation. In this section we describe the simulations done in this context for the Ecuadorian project [6]. 


\subsection{Corsika}

We simulated the development of proton, photon and hadron initiated Extensive Air Showers for the different sites in Ecuador. The strategy used follows a general approach adopted by other LAGO sites for this kind of studies [7]. We considered the primary flux at the top of the atmosphere, taking into account the measured spectra for nuclei with $1 \leq A_{p} \leq 56$. Primary energies $\left(\mathrm{E}_{p}\right)$ were considered from $10 \mathrm{GeV}$ to $10^{6} \mathrm{GeV}$ with zenith angles between $0^{\circ}$ to $60^{\circ}$. Showers were simulated using the Extensive Air Showers package CORSIKA v7.350 [8]. QGSJET II-04 model was used. No thinning was applied, thus all particles above a very small threshold were followed until absorbed in the atmosphere or reached the detector level.

We obtained the secondary particle flux as a function of the particle momentum for the EPN site (LAT $0^{\circ} 12^{\prime} \mathrm{S}$, LONG $78^{\circ} 29^{\prime} \mathrm{O}$ and 2817 m.a.s.l.) The geomagnetic field at this location $\left(\mathrm{B}_{x}=27.09[\mu \mathrm{T}], \mathrm{B}_{z}=11.05[\mu \mathrm{T}]\right)$ was used and geomagnetic effects were taking into account by calculating the rigidity cut-off for the EPN site. We use MAGNETOCOSMICS, a GEANT4 based simulation package, to solve the equations of motion for single particles in the geomagnetic field at this latitude for different arrival directions [9].

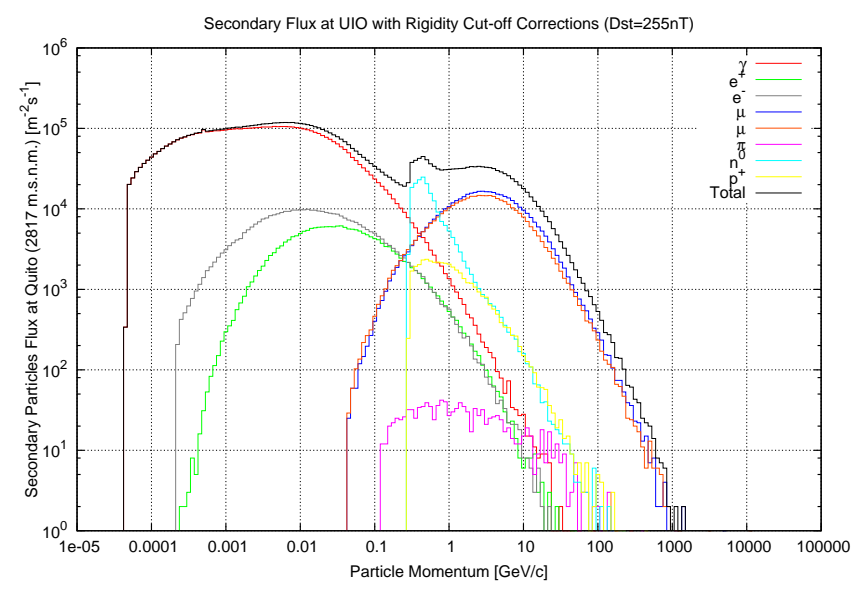

Figure 6: Simulated secondary flux at detector level for "Politanque" WCD at 2817 m.a.s.l. Rigidity Cut-Off corrections were taken into account.

\subsection{Geant4 simulation}

For the simulation we have used the Geant4 release 9.6.p02 [10]. We have modified the simulation included in the examples-optical, named LXe as the base code for the simulation, as well as the B1 example [11].

The world volume is declared as a box filled with "G4_Air", here the main volume is built using the polycone function, the resulting volume is shown in figure 8. The main volume is filled with "G4_Water" and covered with a layer of "G4_POLYETHYLENE". The surface between the polyethylene and the water is declared as an optical surface, here we can modify the properties of the material, for instance, we declared an efficiency between $90 \%$ and $95 \%$ for the reflectivity of the "Tyvek".

The simulation done so far shows the particles created from the interaction of the VEM with the water, the detector response is still under development. 

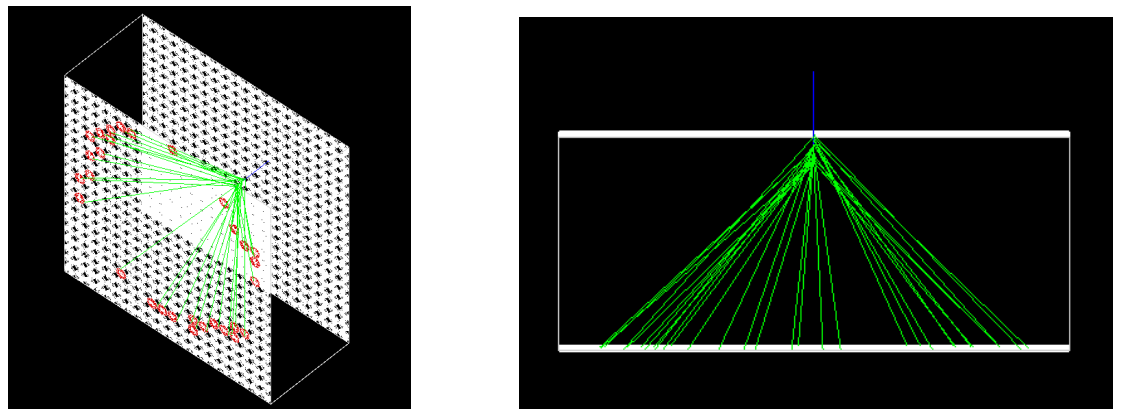

Figure 7: Left: Simulated cherenkov event in the LXe example. Right: Lateral view of the event.
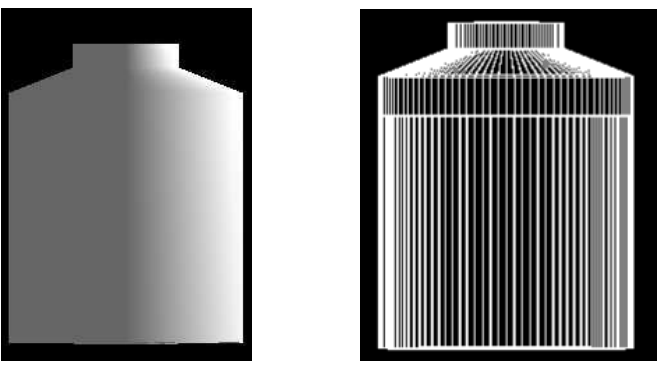

Figure 8: Construction of the main volume shown as solid(left) and wireframe(right).

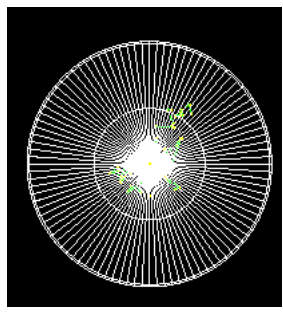

(a)

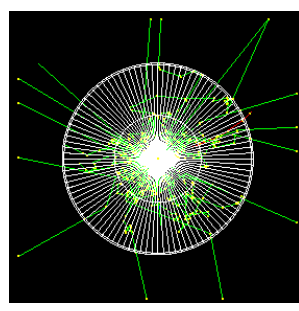

(b)

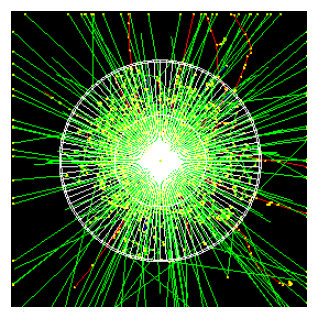

(c)

Figure 9: Development of the VEM in the WCD for an incident: (a) muon 50 [MeV], (b) muon of 1 [GeV], (c) $4000[\mathrm{GeV}]$ muon. The shown view is from above the WCD.

\section{Conclusion}

Data obtained with "politanque" shows the first calibration for the WCD, a longer period of acquisition is needed in order to get a better fit for the first VEM.

Also we found the relationship between the number of events registered and the thresholds for the same exposure times. This is expected because of the characteristic shape of the energy spectrum for Cosmic Rays. For a higher threshold, it's required a more energetic event, events of higher energies have the property that their frequency diminishes.

We have succesfully simulated the secondary flux at detector level for "Politanque" WCD at 2817 m.a.s.l. taking into account the Rigidity Cut-Off using Corsika. We found that the principal contribution in energy of the particles that get to the detector are muons. The simulations in Geant4 are still under development, but the results so far are promising.

The cosmic ray physics community in Ecuador is a growing collaboration. There is a long 
term future plan to install a national detector network in order to take advantage of this unique location. This work shows the first steps toward the realization of this plan.

\section{Acknowledgements:}

The LAGO Collaboration is very thankful to the Pierre Auger Collaboration for its continuous support. The authors of this paper gratefully acknowledge the financial support from Escuela Politécnica Nacional, Universidad San Francisco de Quito USFQ, Fundación CEDIA through CEPRA IX research grants and SENECYT for the prometeo program.

\section{References}

[1] Asorey H. for the LAGO Collaboration. (2015). LAGO: the Latin American Giant Observatory, Proceedings, 34rd International Cosmic Ray Conference (ICRC2015).

[2] Sidelnik, I. for the LAGO Collaboration. (2015). The Sites of the Latin American Giant Observatory, Proceedings, 34rd International Cosmic Ray Conference (ICRC2015).

[3] Díaz M. for the LAGO Collaboration. (2014). Implementing a WCD detector system in Riobamba as part of the LAGO project, Proceedings, 33rd International Cosmic Ray Conference (ICRC2013), Rio de Janeiro, Brazil.

[4] Cotzomi, J., Martínez, O., Moreno, E., Salazar, H., Villaseñor, L. (2005). Air shower array at the university of Puebla for the study of cosmic rays, Revista Mexicana de Física, 51(1), 38-46.

[5] Sánchez, C., Rubén, J. (2015). Instrumentación para el Arreglo de Detectores de Superficie LAGOMéxico, Benemérita Universidad Autónoma De Puebla, PHD Thesis, México.

[6] Mantilla C. et.al. (2015). Implementing a WCD detector system in Ecuador as part of the LAGO Project, Proceedings SILAFAE edition X, Nuclear Physics B Proceedings Supplement, Vol 1.

[7] Asorey H. for the LAGO Collaboration. (2015). The LAGO Solar Project, Proceedings, 34th International Cosmic Ray Conference (ICRC2013), Rio de Janeiro, Brazil.

[8] Heck, D. et. al. (1998). CORSIKA: a Monte Carlo code to simulate extensive air showers, Report FZKA 6019, Forschungszentrum Karlsruhe GmbH.

[9] Agostinelli, S. et. al. (2003). Geant4:a simulation toolkit, Nuclear Instruments and Methods in Physics Research Section A: Accelerators, Spectrometers, Detectors and Associated Equipment, 506(3), 250303.

[10] Geant4 collaboration. (2013). Geant4 User's guide for application developers.

[11] Martínez, A. (2012). Simulación del experimento LAGO en Sierra Negra, Universidad Autónoma del Estado del Hidalgo, Bachelor Thesis, México. 